\title{
Iron heart - the case of non-ischemic cardiomyopathy
}

\author{
Authors: Jagienka Szulc-Bagrowska ${ }^{1}$, Małgorzata Lelonek ${ }^{2}$ (mentor) \\ ${ }^{1}$ Students' Scientific Association by Department of Noninvasive Cardiology, Medical University of \\ Lodz, Lodz, Poland \\ ${ }^{2}$ Department of Noninvasive Cardiology, Medical University of Lodz, Lodz, Poland
}

DOI: https://doi.org/10.26800/LV-142-supp5-29

\section{Background:}

Iron deposition in cardiomyocytes in primary or secondary haemochromatosis can result in the development of dilated cardiomyopathy and heart failure.

\section{Case presentation:}

A 63-year-old man has been admitted to the Noninvasive Cardiology Unit due to exacerbation of chronic heart failure with reduced ejection fraction (HFrEF). The patient was previously hospitalized 10 months ago for the same reason. His history included non-ischaemic dilated cardiomyopathy diagnosed at the age of 58 (no atherosclerotic lesions were found in coronary angiography), insulindependent diabetes mellitus, hypercholesterolemia and two-time pulmonary oedema (in 2016 and 2018). ECG at admission showed atrial fibrillation with rapid ventricular action and single ventricular extrasystoles. Echocardiography revealed atrial and left ventricular enlargement, systolic dysfunction of both ventricles, $\mathrm{EF}=23 \%$, TAPSE $=15 \mathrm{~mm}$, mild tricuspid regurgitation with medium probability of pulmonary hypertension. In laboratory tests ferritin level was $647.4 \mathrm{ng} / \mathrm{ml}$ and TSAT=42.9\%. Abdominal ultrasound did not reveal any abnormalities. Due to high levels of ferritin and nonischaemic background of dilated cardiomyopathy, the haemochromatosis was suspected and cardiac magnetic resonance (CMR) and genetic test for HFE gene mutation was ordered. The CMR confirmed reduced left ventricular systolic function with $E F=20 \%$, but without myocardial hypertrophy, and revealed left ventricular fibrosis of non-ischemic etiology. On the other hand, genetic testing showed heterozygotic mutation of $\mathrm{C} 282 \mathrm{Y}$ in the HFE gene. According to the diagnostic algorithm for haemochromatosis from 2011 AASLD guidelines, the primary haemochromatosis diagnosis was rejected. The overlap of non-genetic causes of secondary siderosis with the carrier state of the pathogenic variant of the HFE gene was considered. The patient was referred for secondary siderosis causes diagnostics.

\section{Conclusion:}

Among the causes of heart failure with repeated hospitalizations per year, storage disorders should be considered. This is particularly relevant if coronary heart disease is excluded as the most common cause of HFrEF and if laboratory test results abnormalities coexist.

Keywords: haemochromatosis, heart failure, non-ischemic cardiomyopathy 